\title{
The LHCb RICH Upgrade
}

\author{
M. P. Blago* on behalf of the LHCb RICH Collaboration \\ University of Cambridge, Cambridge, United Kingdom \\ CERN, Geneva, Switzerland \\ E-mail: michele.piero.blago@cern.ch
}

The LHCb RICH detectors have been operating successfully since 2010 and proven to be an essential element of the experiment thanks to their excellent particle identification performance. During the Long Shutdown II of the LHC in 2019 - 2020, the two detectors will be upgraded in order to maintain their PID performance while operating at significantly increased luminosity, aiming to collect $5 \mathrm{fb}^{-1}$ per year. This will allow to greatly enhance the statistical precision of physics measurements and to advance the search for very rare B- and D-meson decays.

To cope with the challenges of the $40 \mathrm{MHz}$ readout rate and increased occupancy the two detectors will undergo a major upgrade. The Hybrid Photon Detectors will be replaced by multi-anode PMTs together with new front-end electronics capable of reading out every bunch crossing of the LHC. Additionally, the optics of the upstream RICH detector will be modified. Following many tests with particle beams and simulations, the LHCb RICH Upgrade is now in its final stage. The current status of the upgrade will be presented.

The 39th International Conference on High Energy Physics (ICHEP2018)

4-11 July, 2018

Seoul, Korea

${ }^{*}$ Speaker. 


\section{Introduction}

The two Ring Imaging Cherenkov (RICH) detectors are providing particle identification (PID) information crucial for the physics programme of the LHCb Experiment at CERN [1]. During the planned shutdown between 2019 - 2020, LHCb is undergoing a major upgrade. The instantaneous luminosity will be raised by a factor of five to $2 \times 10^{33} \mathrm{~cm}^{-1} \mathrm{~s}^{-2}$ and the read-out rate is going to increase to $40 \mathrm{MHz}$ to match the proton-proton collision rate and allow for a full software trigger. The resulting increase in data acquisition rate will allow for significant improvements in efficiencies, in particular for hadronic decays which is crucial for the study of many rare decays representing the LHCb physics goal [2].

\section{PID performance}

The PID performance is defined by the efficiency to identify a true Kaon as a Kaon and the probability to misidentify a Pion as a Kaon or another, heavier, hadron. Figure 1a uses simulation to compare the RICH performance in different scenarios. The current performance is shown in black, the performance of the current system in the environment of increased luminosity in red, and the performance of the upgraded system in the environment of increased luminosity in green. One can see that due to the detector upgrade measures the current performance is maintained in the environment of higher luminosity and data acquisition rate.

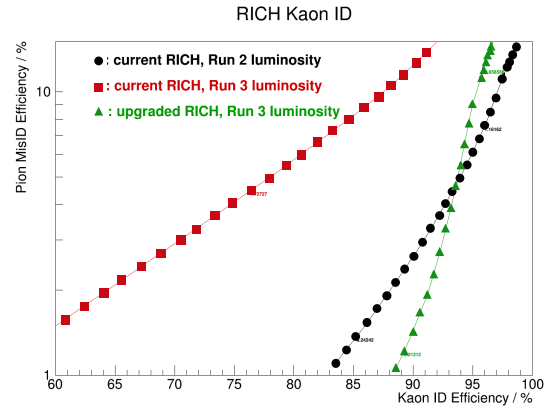

(a)

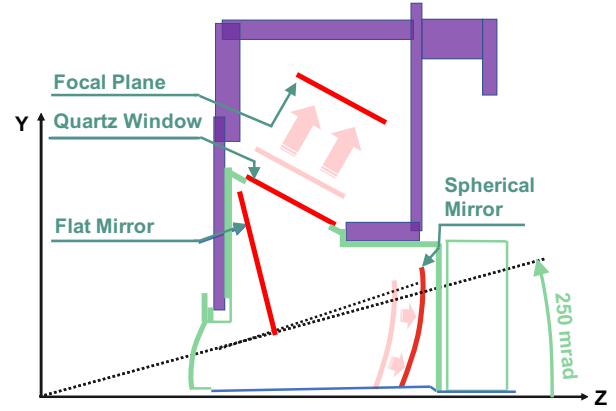

(b)

Figure 1: (a) Simulated efficiency to correctly identify a particle as a Kaon as a function of the probability to misidentify a Pion as a Kaon. (b) Schematic side view of the upper half of the RICH 1 detector after the upgrade with indicated mechanical modifications to increase the focal length.

\section{Photon occupancy}

In order to reduce the occupancy at the detection plane, the new spherical mirrors have an increased focal length compared to the current ones. This requires the repositioning of the mirrors and the move of the detection plane further away as indicated in Figure 1b. To replace the mirrors, their entire support structure will be rebuilt. The spherical mirrors will consist of a light carbon fibre composite material since they are placed inside the LHCb acceptance. Due to the spacial constraints imposed by the magnetic shielding enclosure a compact opto-electronic system is required. 


\section{Photon detection system}

The current photon detectors will be replaced by two types of $8 \times 8$ channel multi-anode photomultiplier tubes (MaPMT) by Hamamatsu ${ }^{1}$ : An 1 inch $\times 1$ inch version for RICH 1 (R13742) and the high-occupancy regions of RICH 2 and a 2 inch $\times 2$ inch version (R13743) for the lowoccupancy regions of RICH 2. The photon detectors and their front-end electronics are combined in modular units called elementary cells, which facilitates assembly and maintenance and allows a similar opto-electronic design for both RICH detectors.

The front-end boards feature custom read-out ASICs called CLARO [3], arranged to ensure a similar signal path length for all channels. The signal is amplified and digitised while the threshold can be set for each channel individually.

The magnetic stray field expected inside the RICH 1 and RICH 2 detectors will reach up to 25 gauss and 5 gauss, respectively. Two options for magnetic shielding were investigated: a full shield surrounding the MaPMTs of the EC and a cross-shield as shown in Figure 2a. Figure $2 \mathrm{~b}$ shows in red the degradation of the MaPMT performance averaged over all its pixels as a function of the magnetic field and in purple the improved performance when using a cross-shaped shield.

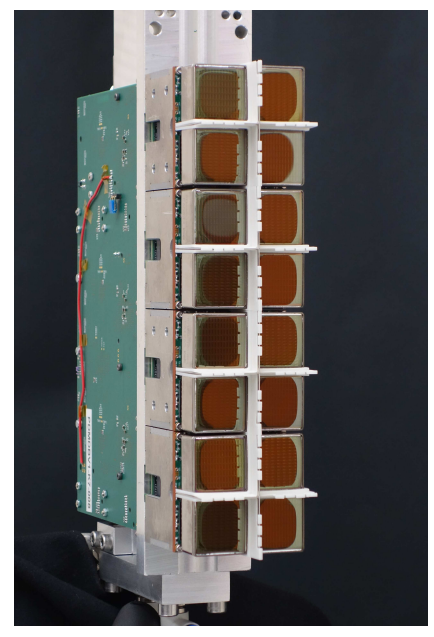

(a)

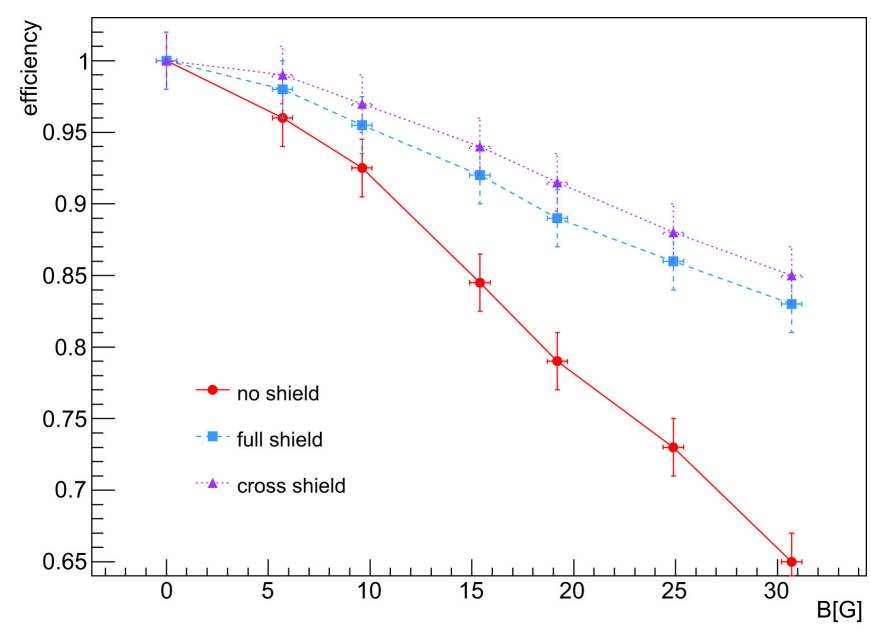

(b)

Figure 2: (a) Photon Detector Module with magnetic cross-shielding. (b) MaPMT performance as a function of the magnetic field strength, recovered by using magnetic shielding. The cross-shield option will be used for the upgrade.

The elementary cells are arranged in Photon Detector Modules (PDM). Each PDM consists of 4 elementary cells with either four $1 \times 1 \mathrm{in}^{2}$ or one $2 \times 2 \mathrm{in}^{2}$ MaPMTs. Figure 2a shows a picture of a PDM with $161 \times 1 \mathrm{in}^{2}$ MaPMTs. The elementary cells are attached to a cooling bar and interfaced to 2 digital boards per PDM, which programme the CLARO ASICs and process the data to transmit it to an online computing farm [2].

\footnotetext{
1www.hamamatsu.com
} 


\section{Cherenkov angle resolution}

The individual contributions to the Cherenkov angle resolution and photon yield are shown in Table 1. The quantum efficiency of the MaPMTs is shifted towards the green wavelength region with respect to the currently used photon detectors which improves the chromatic error of the experiment. The emission point uncertainty is caused by the mirror tilt and the pixel contribution is a result of the detector granularity [4].

\begin{tabular}{|l|c|c|c|c|c|}
\hline \multicolumn{1}{|c|}{ Uncertainties } & Chromatic [mrad] & Emission point [mrad] & Pixel [mrad] & Total [mrad] & Photon Yield \\
\hline RICH 1 (current) & 0.84 & 0.76 & 0.6 & 1.65 & 32 \\
\hline RICH 1 (upgraded) & 0.58 & 0.37 & 0.44 & 0.88 & 42 \\
\hline RICH 2 (current) & 0.48 & 0.27 & 0.19 & 0.76 & 24 \\
\hline RICH 2 (upgraded) & 0.31 & 0.27 & 0.19 & 0.60 & 22 \\
\hline
\end{tabular}

Table 1: Single photon resolutions and photoelectron yields for RICH 1 and RICH 2.

\section{Conclusion}

The preparations for the upgrade of the RICH detectors in the coming years $2019-2020$ are well underway. At the time of writing, all MaPMTs and CLARO ASICs have been delivered by the manufacturer and are currently in quality assurance phase. The individual components of the upgraded system have been studied extensively in beam experiments [5, 6] and laboratory tests [7, 8]. All elements of the Photon Detector Modules and the modifications to the mechanical structure in RICH 1 have been successfully reviewed for production readiness.

\section{References}

[1] LHCb Collaboration, LHCb detector performance, Int. J. Mod. Phys. A 30 (2015) .

[2] LHCb Collaboration, LHCb PID Upgrade Technical Design Report, Tech. Rep. CERN-LHCC-2013-022. LHCB-TDR-014, Nov, 2013.

[3] M. K. Baszczyk et al., CLARO: an ASIC for high rate single photon counting with multi-anode photomultipliers, Journal of Instrumentation 12 (2017) .

[4] S. Easo et al., Overview of LHCb-RICH upgrade, Nuclear Instruments and Methods in Physics Research Section A 876 (2017) 160 .

[5] M. K. Baszczyk et al., Test of the photon detection system for the LHCb RICH Upgrade in a charged particle beam, Journal of Instrumentation 12 (2017) .

[6] P. Carniti et al., Beam test results for the upgraded LHCb RICH optoelectronic readout system, Nuclear Instruments and Methods in Physics Research Section A 876 (2017) 137.

[7] M. Blago, F. Keizer et al., High rate tests of the photon detection system for the LHCb RICH Upgrade, Nuclear Instruments and Methods in Physics Research Section A 876 (2017) 101.

[8] S. Gambetta et al., First results from quality assurance testing of MaPMTs for the LHCb RICH upgrade, Nuclear Instruments and Methods in Physics Research Section A 876 (2017) 206. 